\title{
Surgical outcomes for pediatric congenital lung malformation: 13 years' experience
}

\author{
Mohamed ElShabrawy Saleh ${ }^{1} \cdot$ Hatem Beshir ${ }^{1,2}\left(\mathbb{D} \cdot\right.$ Gehad Awad $^{1} \cdot$ Ahmad EIDerie $^{1} \cdot$ Mohammed Sanad $^{1,3}$
}

Received: 17 January 2020 / Revised: 21 May 2020 / Accepted: 27 May 2020 / Published online: 11 August 2020

(C) Indian Association of Cardiovascular-Thoracic Surgeons 2020

\begin{abstract}
Background Congenital lung malformations (CLM) are a gamut of lesions that originate throughout the embryonic period and manifest in the neonatal or sporadically in the prenatal period, characteristically might stay well for some time, to be found inadvertently or to present with complications. In the 13 years from Jan. 2003 to Dec. 2015, this prospective cohort study included consecutive pediatric patients under 12 years old, who presented either emergently or electively with any variety of CLM. The lesions encompassed in this assortment were congenital lobar emphysema (CLE), congenital pulmonary airway malformation (CPAM), bronchogenic cysts (BC), and bronchopulmonary sequestration (BPS).

Results Sixty-eight pediatric patients with CLM were operated at our institution; 18 CPAMs, 22 CLEs, 19 BPSs (17 intralobar and 2 extralobar), and 9 patients with BCs. The patients' age ranged from 1 to 54 months (mean age of $10.73 \pm 9.73$ months), with overall male gender predominance $(61.76 \%)$. Both CLE and CPAM had a male predominance, while BC and BPS had equivocal gender distribution. CLE patients had the earliest presentation at $2.89 \pm 1.5$ months and congenital cystic adenomatoid malformations $(\mathrm{CCAM})$ had the latest presentation at $21.78 \pm 15.6$ months $(F=15.27, p<0.0001)$. Lobectomy was the commonest procedure performed. Fifty-nine lobectomies were performed (21 LUL, 15 RLL, 14 LLL, 8 RUL, and 1 middle lobectomy). Six cystectomies were performed for BC. Twenty-three cases (33.8\%) had postoperative complications that were mainly significant or prolonged air leak (13.24\%), pneumonia (5.88\%), 3 cases of hemothorax (4.4\%), pulmonary atelectasis in 2 patients $(2.94 \%), 1$ patient developed effusion (1.47\%), and there were 2 mortalities.

Conclusions CLM must be in mind in the differential diagnoses of any case with repeated infection, respiratory distress, or radiological abnormalities. Surgery in the form of lobectomy or lesser resection is generally safe.
\end{abstract}

Keywords Pediatric lung disease $\cdot$ Lung volume reduction surgery $\cdot$ Thoracic surgery $\cdot$ Lung malformations

Electronic supplementary material The online version of this article (https://doi.org/10.1007/s12055-020-00977-2) contains supplementary material, which is available to authorized users.

Hatem Beshir

hatem.beshir@alexmed.edu.eg; hatem_beshir@msn.com

Mohamed ElShabrawy Saleh

mshabrawy2@gmail.com

Gehad Awad

Gehad_awad@yahoo.com

Ahmad ElDerie

ahmadelderie@mans.edu.eg
Mohammed Sanad

mams@mans.edu.eg; mohamedsanad2008@yahoo.com

1

Department of Cardiothoracic Surgery, Faculty of Medicine, Mansoura University, Mansoura, Egypt

2 Department of Cardiothoracic Surgery, Egypt Ministry of Health and Population, Alexandria, Egypt

3 Department of Cardiothoracic Surgery, Egypt Ministry of Health and Population, Nasser Institute for Research and Treatment, Cairo, Egypt 
Table 1 Classification of congenital lung diseases [1]

\begin{tabular}{lc}
\hline A- Tracheal abnormalities & D- Vascular abnormalities \\
1. Tracheal agenesis and atresia & 1- Pulmonary arterial system \\
2. Congenital tracheal stenosis & i- Unilateral absence of main pulmonary artery \\
3. Tracheomalacia & ii- Idiopathic hyperlucent lung syndrome \\
B- Bronchial branching abnormalities & iii- Pulmonary artery sling (dysphagia lusoria). \\
1. Bronchial atresia & iv- Pulmonary arteriovenous fistula (malformation) \\
2. Congenital bronchiectasis & v- pulmonary stenosis \\
3. Tracheobronchomegaly & vi- isolated pulmonary artery aneurysm \\
4. Laryngotracheo-esophageal cleft & 2- Pulmonary venous system \\
5. Tracheoesophageal fistula & i- Anomalous pulmonary venous drainage (partial/ total) \\
6. Broncho-biliary fistula & ii- Scimitar syndrome \\
7. Azygous lobe & iii- Pulmonary vein varix \\
8. Horseshoe lung & iv- Pulmonary vein stenosis \\
9. Bronchopulmonary foregut abnormalities & v- Abnormal number of pulmonary veins \\
$\begin{array}{l}\text { a. Sequestration } \\
\text { b. Bronchogenic cysts }\end{array}$ & \\
C- Lung bud abnormalities & 3- Lymphatic system \\
1. Pulmonary agenesis, aplasia, hypoplasia & i- congenital pulmonary lymphangectasia \\
2.congenital lobar emphysema & 4- Systemic vascular abnormalities \\
3. congenital cystic adenomatoid & i- Arterial: intralobar sequestration \\
malformation & ii- Venous: azygous lobe \\
4. Infantile pulmonary emphysema & \\
6. Congenital parenchymal cysts & \\
7. pulmonary lymphangiectasia & \\
&
\end{tabular}

\section{Background}

Congenital lung malformations (CLM) are an array of lesions (Table 1) that originate throughout the embryonic period and manifest in the neonatal or seldom in the prenatal period, and could stay asymptomatic to be unintentionally discovered or present with complications [1-3]. The frequency of CLM is unclear. Factually, it was projected that the incidence rate was 1 in 20,000 to 1 in 35,000 live births based on prenatal ultrasonography findings. However, with the better availability of prenatal care for pregnant females, recent approximations range amid 1 in 2500 to 1 in 8000 live births [4].

The lesions incorporated in this variety are CLE, BPS, congenital pulmonary airway malformations (CPAM), formerly recognized as congenital cystic adenomatoid malformations (CCAM), and bronchogenic cysts (BC). They result from perturbations in lung and airway embryogenesis. According to the gestational age and the extent within the tracheobronchial tree, the precise type of CLM is set [5].

CLE constitutes $10 \%$ of congenital lung malformations. It usually presents with single lobar alveolar expansion subordinate to bronchomalacia or absent cartilage, along with air trapping and hyperinflation leading to ventilation-perfusion (V/Q) mismatch owing to atelectasis on the ipsilateral or the contralateral side with a mediastinal shift. It has a prevalence rate of 1 in 20,000 to 1 in 30,000 live births and has a male preponderance with a male to female ratio of 3:1. The left upper lobe is affected in 50\% of cases [4]. It can be asymptomatic or display neonatal respiratory distress (RD). Those with the lesion may experience dyspnea or repeated respiratory infection. Congenital heart disease may be found in $15 \%$ of the cases [2].

BPS is an atypical mass of non-functioning lung tissue that formulates throughout prenatal development that is neither attached to the pulmonary arterial blood supply nor connected to the normal bronchial tree, leading to V/Q mismatch. It can form outside (extralobar/ELS) or inside (intralobar/ ILS) the lungs which do not have their pleural covering. The incidence is $0.29 \%$. The lower lobes are commonly affected. The blood supply for a BPS generally originates at the thoracic aorta, although feeding vessels may arise from divisions of the abdominal aorta, such as the celiac artery. Venous drainage of extralobar BPS is systemic, namely to the vena cava, azygous vein, and even straight into the right atrium. Drainage of intralobar BPS is via the pulmonary vasculature to the left atrium. BPS may even ascend within CPAMs in hybrid lesions, which encompass elements of both types of malformations. Infection, heart failure, carcinoma, and bleeding can ensue in the sequestrated lung. Hence, elective resection is recommended in asymptomatic patients with BPS $[2,5]$. 
CCAM or CPAM is the most common malformation of the lower respiratory tract. The yearly estimated incidence is $1: 8300$ to $1: 35,000$ births without sex predilection. It is a hamartomatous lesion containing tissue from different pulmonary origins. It usually affects a single lobe and has no sex predilection. Modified Stocker classification categorized CPAM based on presumed site of development of the malformation into types $0=$ tracheobronchial, $1=$ bronchial/bronchiolar (3-10 cm and lined by ciliated pseudostratified epithelium), 2 = bronchiolar $(0.5-2 \mathrm{~cm}$ and lined by ciliated cuboidal or columnar epithelium), 3 = bronchiolar/alveolar (adenomatoid elements of the distal airway), and 4/5 distal acinar. Lesions regress antenatally in $59 \%$ of cases. Type 4 and type 1 tend to carry around $4 \%$ malignancy risk $[3,5]$.

$\mathrm{BC}$ form as a consequence of anomalous budding of the bronchial tree through embryogenesis (between 4th and 6th weeks), and as such, they are lined by secretory respiratory cuboid or columnar ciliated epithelium. The wall is histologically similar to the normal bronchial tree, including cartilage, elastic fibers, and mucous glands besides smooth muscle. They are usually single and are filled with fluid rich in water, proteinaceous material, blood products, and calcium oxalate, rather than air. It has a prevalence rate of 1 per 68,000 and typically present during the second decade of life. It can occur in the mediastinum (60-90\%) or be intrapulmonary (perihilar or lower lobes) or have uncommon locations (neck, pericardium, transdiaphragmatic, retroperitoneal) [3, 6].

Congenital parenchymal lung malformations (CLM) are scarce. It is difficult to oversee the incidence of such malformations. The yearly incidence of CLM ranges from 30 to 42 cases per 100,000 population. CLM is the cause of 0.06 to $2.2 \%$ of pediatric admissions to general hospitals [6].

Their clinical presentation ranges widely from respiratory failure after birth to asymptomatic adult patients discovered fortuitously, or present with malignant transformation if left undetected or untreated. It is important to recognize these conditions so that appropriate treatment, usually surgical, can be instituted. Histological structures of CLMs have been described in association with rhabdomyosarcoma, pleuropulmonary blastoma, and bronchioalveolar carcinomas [6].

The usual history is variable. All infants with a prenatal diagnosis necessitate postnatal assessment. Patients should be assessed for related ailments. The existence of mass effects is a warning for therapeutic decompression. The threat of pulmonic compression, infection, and malignant degeneration or transformation makes resection domineering, even in asymptomatic patients. Conservative anatomical pulmonary resection in the form of lobectomy or segmentectomy is the mainstay of treatment. It is well endured, and leads to excellent outcomes, either via open thoracotomy or utilizing video-assisted thoracoscopic surgery (VATS) [6].

\section{Aims and objectives}

Herein, we aim to describe the clinical presentation, associated complications, surgical intervention, and outcomes of different congenital lung malformations in the pediatric age group, presenting to our university children's hospital over 13 years.

\section{Methods}

In the 13-year period from Jan 2003 to Dec 2015, our prospective cohort study included 68 consecutive pediatric patients with congenital lung malformation, who were operated at our university hospital, in a developing country.

\section{Inclusion criteria}

This study included all pediatric patients under 12 years old who presented to us either emergently or electively with any variety of CLM.

The exact distribution of our patients' pathology was the following: 18 patients with a congenital cystic adenomatoid (pulmonary) malformation (CCAM/CCPM), 22 patients with CLE, 19 patients with BPS (17 intralobar and 2 extralobar), and 9 patients with $\mathrm{BC}$.

\section{Exclusion criteria}

Adult patients $\geq 18$ years old were excluded from our study. Patients between 12 and 18 years old were not encountered during the study period. Patients undergoing combined procedures as pulmonary and congenital heart surgery were not eligible. Hitherto, none were encountered during the study interval.

\section{Primary endpoint}

The primary endpoint of our study was the site of affection for each pathology.

\section{Secondary endpoints}

The secondary endpoints were patient demographics, clinical presentation, surgery performed, postoperative course, and complications.

Patients manifested with respiratory distress were operated upon proper diagnosis by clinical and radiographic methods. Stable patients were operated electively after proper management of associated chest infection and taking time doing all relevant investigations. These investigations included a chest $\mathrm{x}$-ray, chest computed tomography (CT) for all patients, CT angiography for cases of pulmonary sequestration, and bronchoscopy on occasion (cases of associated infection). 


\section{Anesthetic protocol}

The induction of anesthesia is very crucial during the surgery of CLM. In patients with CLE, crying may induce a pulmonary valve effect, which may upsurge hyperinflation in the affected lobe and intensify mediastinal shift. This may lead to circulatory insufficiency and cardiac arrest.

Inhalation induction of anesthesia was through using oxygen, sevoflurane, or halothane. Gentle ventilation or highfrequency ventilation were used. High-pressure ventilation was not done while waiting for thoracotomy and lung tissue release with airway pressures controlled between 20 and 25 $\mathrm{cmH}_{2} \mathrm{O}$.

Single lumen endotracheal tubes were used for tracheal intubation. Pediatric and neonatal Carlin's tubes were not available at our institution.

\section{Surgical principles}

The surgeon should be scrubbed and ready for thoracotomy before induction. A right or left thoracotomy was the sole surgical access for resection of CLM in childhood. One patient required re-do thoracotomy for treating broncho-pleural fistula. Video-assisted thoracoscopic surgery was not available at that time for the pediatric age at our institution. Anatomical pulmonary resection was the gold standard in most cases of CLM. Anatomical segmentectomy, sequestrectomy, or cystectomy were performed in some cases. All resected specimens were submitted for histopathology (Fig. 1). The patients were followed up for 1 year via the cardiothoracic and pediatric pulmonology departments on an outpatient basis.

Two patients with CLE underwent left anatomical trisegmentectomy for segments $\mathrm{S} 1, \mathrm{~S} 2$, and $\mathrm{S} 3$ to preserve the superior and inferior lingular segments (S4 and S5). Initial dissection involved the hilar release of superior veins, with clear identification of the lingular branch. After the superior pulmonary vein was ligated, dissection of the main pulmonary artery usually revealed two to three anterior apical branches of the left upper lobe (LUL). These were isolated, ligated, and cut. The posterior branch to the LUL was ligated and cut after first dissecting and stapling the LUL bronchus. The lung was ventilated to help delineate the remaining viable lingual, and the lingual sparing lobectomy is finished by cutting and suturing the lung at the demarcation of the lingual and LUL segments.

\section{Statistical analysis}

The statistical analysis was done using SPSS (Statistical Package for Social Sciences) version 22.0 statistical analysis software (IBM Inc., Chicago, IL). Qualitative data were described using the number and percent. Quantitative data were described using minimum and maximum, mean and standard deviation and median.

As four independent groups were compared, one-way analysis of variance (ANOVA) was used to compare between means of the groups (using the F distribution). Homogeneity of variance, was tested by Leven's test. Means plots were obtained. The significance of the obtained results was judged at the $5 \%$ level.

A comparison between different groups regarding categorical variables was tested using the Chi-square test. When more than $20 \%$ of the cells have expected count less than 5 , correction for chi-square was conducted using Fisher's Exact test or Monte Carlo correction.

\section{Results}

Our study included 68 patients who had any of the congenital lung malformations, classified as follows: 22 patients with lobar emphysema (CLE) (32.35\%), 19 patients with BPS (27.4\%) (2 of these were extralobar), 18 with CPAM (26.47\%), and 9 with bronchogenic cyst (BC) (13.24\%).

Our patients' age ranged from 1 to 54 months (mean age of $10.73 \pm 9.73$ months). A one-way ANOVA was conducted between subjects to compare the mean age between the four subgroups. There was a significant difference at $p<0.05$ level between the four groups for the age at the presentation at which the mean ages in months were $2.89 \pm 1.5$ for CLE (the earliest presenter), $11.67 \pm 6.1$ for CPAM, 21.78 \pm 15.6 for BC (the latest presenter), and $15.53 \pm 7.5$ for BPS. Post hoc comparisons using the Tukey's honestly significant difference (HSD) test indicated that the CLE patients had the earliest presentation and CCAM had the latest presentation $[F(3$, $64)=15.27, \mathrm{MSB}=869.4, \mathrm{MSW}=56.93, P<0.0001)$.

The gender difference was not statistically significant using a Fisher exact test of independence and Monte Carlo correction, though overall $61.76 \%$ of the total patients were males and $38.23 \%$ were females. Both CLE and CPAM had a male predominance, while $\mathrm{BC}$ and BPS had equivocal gender distribution.

The principal presenting symptom was repeated chest infections in 38 patients $(55.89 \%)$ with a statistical significance confirmed by fisher exact test of independence and Monte Carlo correction FE $=132.48$, Mc $p<.0001,95 \%$ CI $[0.001$, $0.43]$. Respiratory distress was the main presenting symptom in 15 patients $(22.06 \%)$; most of these were lobar emphysema patients (14). Patients with CPAM and BPS exhibited high rates of infection. Seven patients $(10.29 \%)$ were asymptomatic and discovered accidentally. The clinical picture of our sample is represented in Table 2.

The particular details of our sample for each type of CLM are illustrated in Table 2 and supplementary Tables 1-4. 

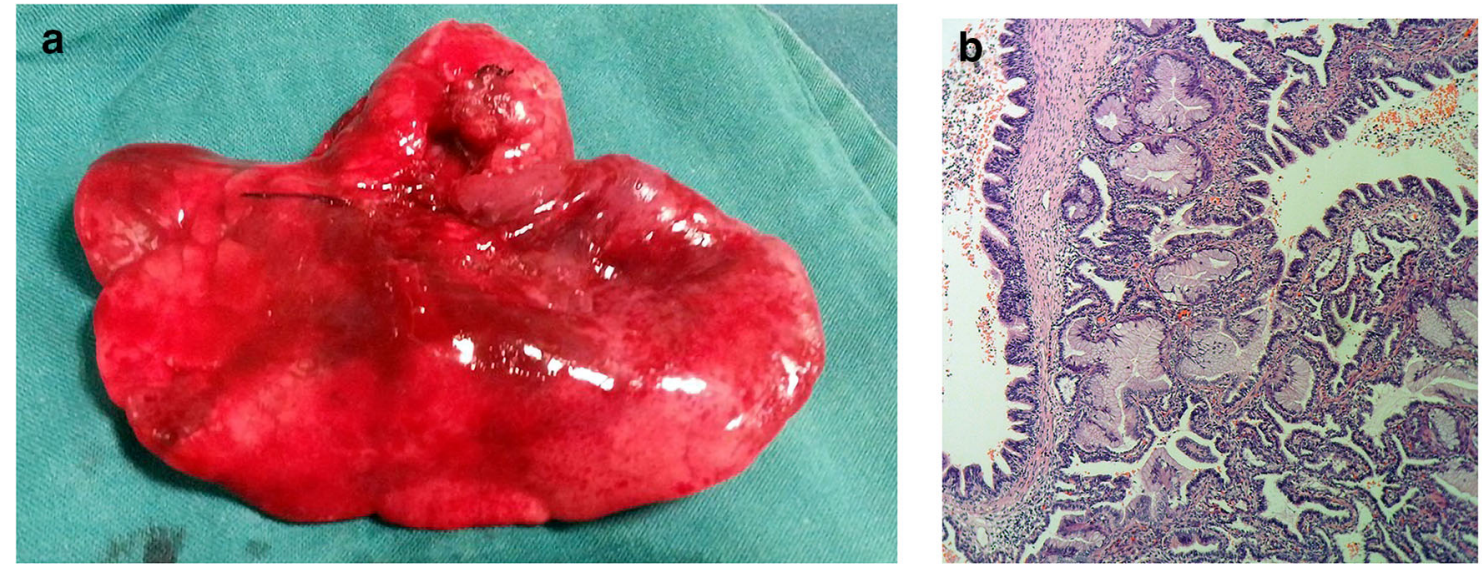

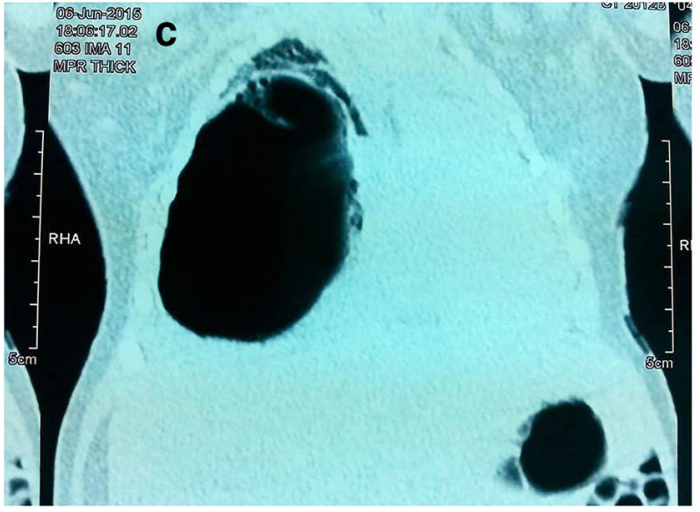

Fig. 1 Congenital cystic adenomatoid malformation (CPAM). a Specimen of right upper lobectomy for CPAM. b Low power light microscopy for Hematoxylin and Eosin film for the excised specimen exhibiting characteristic sawtooth lining with pseudostratified

The LUL were significantly predominant as a common site of CLM (33.8\%) $(\mathrm{FE}=73.25, p<.0001)$, especially in CLE. The right lower lobes (RLL) were the second to be affected (22\%), particularly in CPAM and BPS. The left lower lobes (LLL) were the third common sites $(20.5 \%)$ with a high incidence in BPS. Bronchogenic cysts were almost exclusively located in the mediastinum.

Anatomical resections were performed in all cases. The affected lobes and the type of surgery in each pathology are shown in Table 2 and in the supplementary Tables 1-4.

Lobectomy was the commonest procedure performed. Fifty-nine lobectomies were performed (21 LUL, 15 RLL, 14 LLL, 8 RUL, and 1 middle lobectomy). Left upper lobectomy was the most common procedure in our sample ( $\mathrm{FE}=$ 73.25, $\mathrm{df}=24, p<.0001,95 \mathrm{CI}[0.001,0.042])$. Cystectomy was performed in six patients with $\mathrm{BC}(8.82 \%$ of total procedures) (Table 2) and (supplementary Tables 1-4).

In 18 cases with BPS, lobectomy was performed for 17 patients. Sequestrectomy was performed for 2 patients with extralobar BPS (2.94\%). Two lingular-sparing left upper lobectomies (anatomical trisegmentectomies for segments 1, 2, and 3) were performed for two patients with CLE, as the lingulae were voluminous and unaffected. The feeding vessels for the

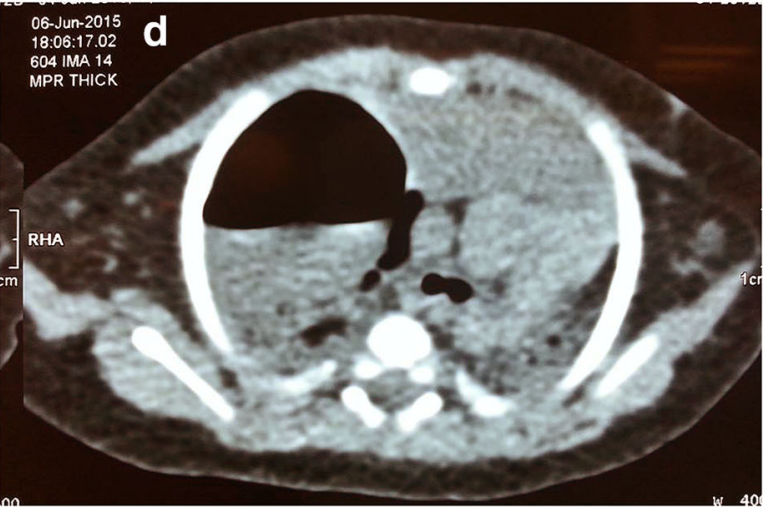

epithelium and mucous cells, consistent with type 1 CPAM. c Saggital computed chest tomography cut showing a large cyst of the case. d Computed tomography coronal cut for the same case

sequestrated lobes were the descending aorta $(94.4 \%)$ and one from the left internal mammary artery $(5.6 \%)(\mathrm{FE}=69.31, \mathrm{df}=$ $9, p<.001$ ) (Table 2) and (supplementary Table 4$)$.

Bronchogenic cysts were the least cases being only nine; five of them were mediastinal, three were intralobar, and the remaining patient had one mediastinal and one in the LUL. The location of mediastinal bronchogenic cysts was subcarinal (4), and paratracheal (2). They showed many presentations in the form of three with a chest infection, two wheezy chests, one with dysphagia, one was tachypneic, and the other two were discovered accidentally. For patients with mediastinal cysts, cystectomy was the surgical procedure while lobectomy was the procedure in cases of intralobar cysts.

The patient with two bronchogenic cysts was not diagnosed preoperatively, so we suspected that the posterior mediastinal mass (as diagnosed preoperatively) was assumed to be the cause of such LUL cyst by bronchial compression. We expected resolution of such cyst after excision of the posterior mediastinal mass. Rather, infection of the cyst occurred ending in cough, expectoration, and respiratory distress. Lobectomy solved the problem but the patient came back to the hospital 1 week after discharge with pneumonia in the LLL. He was treated uneventfully. 
Table 2 Clinical picture of the studied sample

\begin{tabular}{|c|c|c|c|c|c|c|c|}
\hline \multicolumn{2}{|l|}{ Lobe affected } & $\begin{array}{l}\text { Emphysema } \\
\text { (CLE) } n=22\end{array}$ & $\begin{array}{l}\text { CPAM } \\
n=18\end{array}$ & $\begin{array}{l}\text { Bronchogenic cyst } \\
\text { (BC) } n=9\end{array}$ & $\begin{array}{l}\text { Sequestrated lobe } \\
\text { (BPS) } n=19\end{array}$ & Total $n=68$ & Significance \\
\hline \multirow[t]{3}{*}{ Age (months) } & Mean $\pm \mathrm{SD}$ & $2.89 \pm 1.5$ & $11.67 \pm 6.1$ & $21.78 \pm 15.6$ & $15.53 \pm 7.5$ & $10.69 \pm 9.66$ & $p<.0001 *$ \\
\hline & Median & 2.5 & 11 & 18 & 13 & 8 & $F(3,64)=15.27$ \\
\hline & IQR & $1-6$ & $4-24$ & $6-54$ & $5-33$ & $1-54$ & $\mathrm{MSB}=869.4, \mathrm{MSW}=56.93$ \\
\hline \multirow[t]{2}{*}{ Sex } & Male & $16(72.72)$ & $11(61.11)$ & $5(55.56)$ & $10(52.63)$ & $42(61.76)$ & $\mathrm{FE}=2.04, \mathrm{df}=3$ \\
\hline & Female & $6(27.27)$ & $7(38.89)$ & $4(44.44)$ & $9(47.37)$ & $26(38.23)$ & $p=0.58$ N.S. \\
\hline \multicolumn{8}{|c|}{ Presenting symptoms } \\
\hline \multicolumn{2}{|l|}{ None } & - & $3(16.67)$ & $2(22.22)$ & $2(10.53)$ & $7(10.29)$ & $\mathrm{FE}=132.48$ \\
\hline \multicolumn{2}{|l|}{ R.D. } & $14(63.64)$ & - & $1(11.11)$ & - & $15(22.06)$ & $\operatorname{Mc} p<.0001 *$ \\
\hline \multicolumn{2}{|l|}{ Wheezes } & - & - & $2(22.22)$ & - & $2(2.94)$ & $95 \%$ CI $[0.001,0.43]$ \\
\hline \multicolumn{2}{|l|}{ Infection } & $5(7.35)$ & $15(83.33)$ & $3(33.33)$ & $15(78.95)$ & $38(55.89)$ & \\
\hline \multicolumn{2}{|l|}{ Dysphagia } & - & - & $1(11.11)$ & - & $1(1.47)$ & \\
\hline \multicolumn{2}{|l|}{ F.T.T. } & $3(13.63)$ & - & - & - & $3(4.41)$ & \\
\hline \multirow{2}{*}{\multicolumn{2}{|c|}{ Hemoptysis }} & - & - & - & $2(10.53)$ & $2(2.94)$ & \\
\hline & & \multicolumn{6}{|c|}{ Radiological findings } \\
\hline \multicolumn{2}{|c|}{ Pneumothorax } & 13 & 3 & 2 & - & 18 & $\mathrm{FE}=10.55$ \\
\hline \multicolumn{2}{|c|}{ Pneumomediastinum } & 5 & 1 & 9 & - & 15 & $\operatorname{Mc} p=.103$ \\
\hline \multicolumn{2}{|c|}{ S.C. emphysema } & 3 & 1 & 1 & - & 5 & \\
\hline \multicolumn{7}{|c|}{ Lobe/ Site of pathology and surgery performed } & $\mathrm{FE}=73.25, p<.0001 *$ \\
\hline \multicolumn{2}{|l|}{ RUL } & $5(22.73)$ & $2(11.11)$ & $1(11.11)$ & - & $8(11.7 \%)$ & 8 RU lobectomy \\
\hline \multicolumn{2}{|l|}{ RLL } & - & $6(33.33)$ & $1(11.11)$ & $8(42.11)$ & $15(22 \%)$ & 15 RL lobectomies \\
\hline \multicolumn{2}{|l|}{ ML } & - & $1(5.56)$ & - & - & $1(1.47 \%)$ & One ML \\
\hline \multicolumn{2}{|l|}{ LUL } & $17(77.27)$ & $4(22.22)$ & $1(11.11)$ & $1(5.26)$ & $23(33.8 \%)$ & $\begin{array}{l}21 \text { LU lobectomy } \\
2 \text { (CLE) Segmentectomies }\end{array}$ \\
\hline \multicolumn{2}{|l|}{ LLL } & - & $5(27.78)$ & $1(11.11)$ & $8(42.11)$ & $14(20.5 \%)$ & 14 LL lobectomies \\
\hline \multicolumn{2}{|l|}{ Mediastinum } & - & - & $6(66.67)$ & - & $6(8.82)$ & 6 cystectomies \\
\hline \multicolumn{2}{|l|}{ Extralobar } & - & - & - & $2(10.53)$ & $2(2.94)$ & 2 sequestrectomy \\
\hline
\end{tabular}

Postoperative course and complications

\begin{tabular}{|c|c|c|c|c|c|c|}
\hline & Mean $\pm \mathrm{SD} / \mathrm{IQR}$ & Mean $\pm \mathrm{SD} / \mathrm{IQR}$ & Mean $\pm \mathrm{SD} / \mathrm{IQR}$ & Mean $\pm \mathrm{SD} / \mathrm{IQR}$ & Mean $\pm \mathrm{SD} / \mathrm{IQR}$ & Significance \\
\hline \multirow[t]{2}{*}{ ICU stay (days) } & $2.18 \pm 1.7$ & $2.89 \pm 2.67$ & $1.86 \pm 1.01$ & $1.95 \pm 1.22$ & $2.27 \pm 1.87$ & $p=.41, \mathrm{df}=3,62$ \\
\hline & $1-7$ & $1-12$ & $1-4$ & $1-6$ & $1-12$ & $F=0.98$ \\
\hline \multirow[t]{2}{*}{ Drain time (days) } & $3.27 \pm 1.61$ & $3.94 \pm 2.47$ & $4.14 \pm 1.35$ & $4.05 \pm 1.43$ & $3.77 \pm 1.81$ & $p=.47, \mathrm{df}=3,62$ \\
\hline & $2-7$ & $2-12$ & $2-6$ & $2-7$ & $2-12$ & $F=0.85$ \\
\hline \multirow[t]{2}{*}{ Hospital stay (days) } & $5 \pm 2.65$ & $6.67 \pm 3.27$ & $6.43 \pm 1.62$ & $6.63 \pm 1.77$ & $6.08 \pm 2.6$ & $p=.13, \mathrm{df}=3,62$ \\
\hline & $3-14$ & $3-16$ & $4-9$ & $4-10$ & $3-16$ & $F=1.98$ \\
\hline Complications & $n(\%)$ & $n(\%)$ & $n(\%)$ & $n(\%)$ & $n(\%)$ & \\
\hline Air leak & $3(13.64)$ & $3(16.67)$ & $1(11.11)$ & $2(10.53)$ & $9(13.24)$ & $p=.25$ \\
\hline Atelectasis & - & - & $1(11.11)$ & $1(5.26)$ & $2(2.94)$ & \\
\hline $\begin{array}{l}\text { Broncho-pleural fistula- } \\
\text { mortality }\end{array}$ & - & $1(5.56)$ & - & - & $1(1.47)$ & \\
\hline Effusion & - & $1(5.56)$ & - & - & $1(1.47)$ & \\
\hline Hemothorax & - & $2(11.11)$ & - & $1(5.26)$ & $3(4.41)$ & \\
\hline Intraoperative bleeding & - & - & - & $1(5.26)$ & $1(1.47)$ & \\
\hline $\begin{array}{l}\text { Intraoperative thoracic duct } \\
\text { injury }\end{array}$ & - & - & $1(11.11)$ & - & $1(1.47)$ & \\
\hline $\begin{array}{l}\text { Mechanical } \\
\text { ventilation-mortality }\end{array}$ & $1(4.55)$ & - & - & - & $1(1.47)$ & \\
\hline Pneumonia & $1(4.55)$ & $2(11.11)$ & - & - & $3(4.41)$ & \\
\hline Pneumonia post discharge & - & - & $1(11.11)$ & - & $1(1.47)$ & \\
\hline Total complications & $3(13.04)$ & $9(39.13)$ & $4(17.39)$ & $5(21.74)$ & $23(33.82)$ & \\
\hline
\end{tabular}

One patient had two surgeries for excision of mediastinal bronchogenic cyst and left upper lobectomy for the same pathology

$C L E$ congenital lobar emphysema, CPAM congenital pulmonary airway malformation, $B C$ bronchogenic cyst, $B P S$ sequestrated lobe/bronchopulmonary sequestration, $R D$ respiratory distress, FTT failure to thrive, S.C subcutaneous, $L U L$ left upper lobe, $R U L$ right upper lobe, $M L$ right middle lobe, $L L L$ left lower lobe, $R L L$ right lower lobe

$n$ sample size, \% percentage, $S D$ standard deviation, $I Q R$ interquartile range, $F$ statistic for ANOVA, $M S W$ mean square within groups, $M S B$ mean square between groups, $F E$ Fisher exact test for independence, $M c$ Monte Carlo correction for cell counts less than $5, \chi^{2}$ chi square, $p p$ value, $d f$ differential, $C I$ confidence interval

* Statistical significance at $p<.05$ 
One patient had two procedures for excision of the mediastinal bronchogenic cyst and left upper lobectomy for the same pathology in the same setting (Table 2) and (supplementary Table 3 ).

Twenty-three cases $(33.8 \%)$ had various complications without statistical significance. Postoperative complications were significant or prolonged air leak (13.24\%), pneumonia $(5.88 \%)$, three cases of hemothorax (4.4\%), pulmonary atelectasis in two patients (2.94\%), and one patient developed effusion (1.47\%). There were two mortalities (Table 2) and (supplementary Tables 1-4).

Two intraoperative complications were encountered in the form of an injury to the thoracic duct that was mass-ligated and another case with injury to the systemic feeding artery from the descending thoracic aorta to the sequestrated lobe, while dissecting the inferior pulmonary ligament. The feeding vessel was not evident in the $\mathrm{CT}$ scan and was controlled using vascular clamps without significant blood loss.

Two mortalities were recorded (2.94\%). The first was an 18month-old male child who presented with repeated chest infections and was diagnosed with LLL CPAM. His surgery was postponed many times due to persistent infection. Repeated bronchoscopy revealed hyperemia and pus. Surgery was done after aggressive medical treatment. He underwent left lower lobectomy and excision of a wedge from the lingula in the proper plane. After 3 days, he developed an increasing air leak and lung collapse. The presence of bronchopleural fistula (BPF) was confirmed by bronchoscopy. He needed re-exploration after 1 week with reinforced closure of the bronchial stump by a pleural flap. He was discharged from intensive care unit (ICU) on day 12 but unfortunately succumbed on day 16 postoperatively after developing a fistula again and died from aspiration pneumonia (supplementary Table 2).

The second mortality was a 3.5-month-old male, who presented with infection and was diagnosed with CLE; prolonged mechanical ventilation was required and mortality was recorded at day 5 postoperatively (supplementary Table 1 ).

Our strategy was to admit the patient in the ICU for a minimum of $24 \mathrm{~h}$. The ICU stay for all patients ranged from 1 to 12 days with a mean of $2.27 \pm 1.87$ days. The drainage time varied from 2 to 12 days with a mean of $3.77 \pm 1.81$. The postoperative hospital stays ranged from 3 to 16 days with a mean of $6.08 \pm 2.6$ days. ICU stay, drainage time, and hospital stays (in days) were statistically insignificant among the four groups of patients (Table 2) and (supplementary Tables 1-4).

\section{Discussion}

Congenital lung malformations are rare anomalies whose appropriate diagnosis and management mirror the degree of general healthcare of the community. To be estimated properly, it needs prenatal diagnosis and management in many cases. In developing countries, many patients with CLM are diagnosed rarely antenatally and may be wrongly diagnosed postnatally as infectious lung diseases. Many cases are revealed at autopsy.

Being a tertiary referral center, our university hospital is the medical destination for many neighboring governorates. Our experience over this extended time (13 years) included 68 patients.

Our patient's age ranged from 1 to 54 months (mean age was $10.73 \pm 9.73$ months). Of these, $61.7 \%$ were males. A retrospective study by Guo et al. [7] performed over 9 years, included 96 patients diagnosed with congenital cystic lung lesions aged from 4 days to 13 years with equal male to female distribution. Ferreira et al. [8] described a mean age at the time of surgery of 31 months (median, 17 months; range 1120 months) and a male sex predominance (60\%).

Our 68 patients were divided as follow: 22 patients (32.3\%) with CLE, 19 patients (28\%) with BPS (2 extralobar), 18 (26.5\%) with CPAM, and $9(13.2 \%)$ with BC. In a similar study done by Costa Junior et al. 2008, [9] on 60 patients, the distribution was as follows: BC 45\%, 23.33\% cases of CLE, $16.67 \%$ BPS, and $15 \%$ cases of CPAM. Ferreira et al. [8] too showed nearly a similar distribution to us: $37 \%$ lobar emphysema, $22.85 \%$ CPAM, and $22.85 \%$ sequestration. Guo et al. [7] described the distribution of CPAM (42\%), BPS (13\%), BC (13\%), CLE (3\%), and unclassified congenital cystic lung lesions with necrotizing pneumonia-like picture (30\%). Only $34.32 \%$ of the cases were operated during that study.

Saeed et al. [10] reported 38 children with CLM in an 11year study. Thoracotomy and lobectomy/excision was done in 23 , and 15 had thoracoscopic lobectomy/excision. The mean age of operation was 18 months (range 2 days to 96 months). Twenty patients had signs of recurring preoperative infection with pleural adhesions and hilar thickening, mandating conversion of 10 thoracoscopic cases to thoracotomy. Histology established a diagnosis of 68.4\% CPAMs, 5.3\% hybrid lesions, $18.42 \%$ sequestrations, and $7.89 \%$ bronchopulmonary malformations.

The most affected lobe was the LUL (33.8\%), followed by the RLL (22\%), LLL (20.5\%), RUL (11.7\%), and lastly the middle lobe (ML) in one patient (1.47\%). Such distribution does not differ largely from that of Ferreira et al. [8] which was LUL $37 \%$, LLL $20 \%$, RUL $20 \%$, RLL $17 \%$, and ML5.7\%.

\section{Congenital lobar emphysema}

As regards lobar emphysema, we had 22 patients, 16 of them were males $(72.7 \%)$. The LUL was affected in 17 patients (77.2\%) and the remaining were in the RUL. Their ages ranged from 1 to 6 months. Lobectomy was the procedure done for all patients except 2 , who were subjected to left upper segmentectomy with preservation of the lingula.

The main complaint was respiratory distress (14), followed by repeated chest infection (5) and failure to thrive (3). 
The diagnoses of lobar emphysema were distinctly the seamless ones. Patients normally presented early with respiratory distress that necessitated hospitalization. Chest X-ray (CXR) was almost typical with emphysematous lobe, mainly the LUL, causing a contralateral mediastinal shift. It is very important to auscultate the chest. In the case of CLE, there is diminished air entry over the emphysematous lobe. If such air entry is good, compensatory emphysema should be suspected. In stable patients, fiberoptic bronchoscopy can be used for the removal of obstructing mucous plug or any other intrabronchial pathology.

The pathophysiology of these patients resembles those with tension pneumothorax; some cases may collapse during induction of anesthesia. Our strategy during the induction of anesthesia was to leave the patient to breathe spontaneously and to avoid manual ventilation. The surgeon should be scrubbed and prepared. We encountered one patient who developed progressive hypoxia after induction of anesthesia that deteriorated with manual ventilation. Positioning and scrubbing the patient within no time followed by rapid thoracotomy and delivery of the diseased lobe outside the chest improved the situation.

In all cases of lobar emphysema, the remaining lobe, which was usually the LUL, seemed initially too small to fill the left hemithorax. However, after the inflation of this lobe, compensatory mechanisms like diaphragmatic elevation, mediastinal shift, and narrowing of the intercostal spaces solved this problem within the early postoperative period. At the end of the maneuver, while taking the peri-costal sutures, additional sutures were inserted around remote ribs, two above and two below or more ribs, to attain rib crowding.

In two patients, the lower lobe was too small and the lingula appeared healthy and voluminous, so we decided it was beneficial to do left upper segmentectomy with preservation of the lingula; the patients had an uneventful course.

The ICU stay ranged from 1 to 7 days. The chest tube was removed after 2 to 7 days. The total hospital stay ranged from 3 to 14 days.

Only three patients showed significant air leaks with longer ICU and hospital stay. One patient developed hospital-acquired pneumonia; he was discharged after 2 weeks in a good condition. One patient showed an unfavorable postoperative course and he was ventilatordependent. The cause was an imperfect surgical technique and wrong decision about the diseased lobe. The operating surgical team of that case was not an expert in this type of surgery. The patient died after 5 days.

\section{Congenital pulmonary airway malformations}

CPAM were 18 (26\%); CPAM comes in the third order after lobar emphysema and sequestration, which may be underestimated. The patient usually presents with repeated chest infections. In our country, due to incorrect diagnosis of bronchial asthma, many cases were not diagnosed until they became adult or developed complications. Fifteen patients $(83.3 \%)$ presented with a chest infection while 3 were discovered accidentally. CPAMs represented $51.2 \%$ of all patients in the study of Mullassery et al. [11].

Most of other series included a significant number of neonatal and even prenatal cases, while our patients' ages ranged from 4 to 24 months, and there were no neonatal cases. This may reflect the lack of either diagnosis or proper surgical management for such cases. Twenty-four percent of cases of CPAM in the study of Karunasumetta et al. [12] had prenatal diagnosis.

\section{Bronchopulmonary sequestration}

The diagnosis of cases of sequestration requires experienced doctors, who are aware of such anomaly and proceed with CT angiography for confirmation of the disease. Our patients presented with repeated chest infections in 15 patients (79\%) and blood-tinged sputum in 2 cases $(10.5 \%)$. The other 2 patients were relatively older (33 m and $23 \mathrm{~m}$ female patients) and were incidentally discovered.

Azizkhan et al. [13] found that patients who are asymptomatic at birth can subsequently develop symptoms like cough, hemoptysis, and mainly recurrent pneumonia. Savic et al. [14] in their collective series of 540 patients found $15 \%$ of patients remained asymptomatic and were incidentally diagnosed.

We have only one patient whose affected lobe was the left upper and the feeding vessel was originating from the internal mammary artery. Gezer et al. [15] and Jesch et al. [16] reported systemic feeding arteries, rather than the aorta, in $15 \%$ cases.

In the other 18 cases, the affected lobe was either the right or left lower lobe with a systemic feeding artery from the descending aorta, either the thoracic or abdominal. We had only 2 extralobar sequestrations $(10.5 \%)$. The ratio between intralobar pulmonary sequestration and extralobar pulmonary sequestration was 3:1 in the study of Corbett et al. [17].

We had no mortality in the pulmonary sequestration cases. One patient had intraoperative bleeding from the systemic feeding vessel, but it was controlled successfully. Savic et al. 1979 [14] reported that, among 540 pulmonary resections for pulmonary sequestration, there were only 5 deaths due to uncontrollable hemorrhage intraoperatively.

\section{Bronchogenic cysts}

Maier [18] classified bronchogenic cysts into five groups by location: paratracheal and miscellaneous including pericardial, cervical, subcutaneous, and abdominal sites. Up to $1 / 3$ of bronchogenic cysts remain asymptomatic $[18,19]$. 
Conservative management of an asymptomatic patient may be offered. However, most prefer to excise the lesion due to fear of expansion, infection, or hemorrhage. Few case reports of malignant alteration have been reported. Since most BCs are linked to the proximal bronchial tree, resection sans loss of substantial lung tissue is conceivable. A diversity of $\mathrm{BC}$, that results in the formation of multiple cystic structures within the peripheral lung parenchyma (also recognized as bronchiolar cysts or cystic bronchiectasis), is allied to recurring infections and resection is endorsed [20]

\section{Postoperative complications}

Our sample carries a $33.8 \%$ complication rate (Table 2 ). This can be attributed to a large number of cases with air leak (13.24\%) with all grades (significant or prolonged) that were included as a complication in our sample. The mean drainage times were $3.77 \pm 1.81$ (IQR 2-12) days before the drains were removed or one patient died of fistulation. Two mortalities were recorded.

Stanton et al. [20] performed a systematic review and metaanalysis that involved a series of 41 reports describing 1070 patients (of whom $79 \%$ were antenatally detected), published between 1996 and 2008, where the postnatal management of congenital cystic lung lesions was designated. Five hundred five neonates endured without surgery into infancy, of whom only $3.2 \%$ became symptomatic. For all ages, elective surgery was allied to significantly fewer complications than emergency surgery. The risk ratio was 2.8 (95\% CI 1.4-5.5; $p<.005)$. Complications of emergency neonatal surgery were reported in 23 series and occurred in 26/93 (28\%) of cases; air leak $(12 \%)$, infection $(6.5 \%)$, bleeding $(1 \%)$, and effusion $(1 \%)$. Late complications were reported in $10 \%$ including residual disease $(3 \%)$, infection $(3 \%)$, bronchiolitis $(2 \%)$, respiratory distress syndrome (2\%), infection (1\%), and asthma $(1 \%)$. Eight patients died after emergency neonatal surgery $(7.5 \%)$.

Complications occurred in $2 / 22$ cases $(9 \%)$ of elective neonatal surgical cases. Early complications were air leak $(4.5 \%)$ and effusion (4.5\%). No late complications were reported, and no deaths occurred.

There were 29 reports ( 871 cases) that designated 505 (58\%) asymptomatic antenatally diagnosed cases, who endured the perinatal period without surgery into infancy. Of these, $505(3.2 \%)$ turned into symptomatic at a median age of 6.9 months (range, $2.5-10$ months; 4 reports). Overall, the median age at which infants developed symptoms was 10 months (range, 2 months to 8.5 years; 13 series).

Emergency surgery for symptomatic cases was done in 158 infants. The median age at surgery was 56 months (range, 2 months to 8.5 years; 12 series).

Complications were specified in $23(17 \%)$ of infant symptomatic cases. Primary complications (13\%) were air leak $(4 \%)$, effusion $(2 \%)$, pneumonia $(1.5 \%)$, atelectasis $(1.5 \%)$, bleeding, wound infection, respiratory failure, and esophageal leak $(0.7 \%$ each). Late complications $(4 \%)$ were asthma $(2 \%)$, recurrent pneumonia $(0.7 \%)$, and residual disease $(0.7 \%)$. Four infants $(3 \%)$ died after infant emergency surgery. Elective surgery was carried out in 436 infants.

Complications of elective infant surgery occurred in 16 $(5 \%)$ of cases. Early complications (20 cases, 6\%) were air leak $(3 \%)$, infection $(0.6 \%)$, effusion $(0.3 \%)$, and retained sponge $(0.3 \%)$. Late complications were residual disease $(0.6 \%)$ and asthma $(0.3 \%)$. One infant $(0.3 \%)$ died after elective surgery.

The type of surgery as specified in 328 cases was lobectomy in 268 and segmentectomy in 60. Residual disease was reported as a complication in a total of 9 patients (15\%) who had experienced segmental resection. No residual disease was reported following lobectomy. Complete resolution of antenatally diagnosed lesions was reported in a total of 49 cases (11 series) [19].

\section{Timing for surgery and surgery for asymptomatic patients}

The proper time for surgery is still a matter of debate. The management of asymptomatic patients is controversial. Some authors advocate close observation, whereas others favor elective resection in every case because of the risks of recurrent infection or malignant transformation. One of the pros for early resection of asymptomatic CLMs is that this may allow better compensatory lung than later resections.

Lung development and growth are thought to endure into childhood and even young adult life via an upsurge in the number of alveolar units (that continues till 2 years of age) with the rest being due to an extension in size of remaining alveoli.

Few studies have endeavored to report on this issue [19-23]. In 1998, Nakajima et al. [21] settled that resection at age less than 4 years was related to better pulmonary function. Similarly, Komori et al. [22] and Marshall et al. [23] have concluded that surgery earlier than 1 year of age is preferable $[19,20]$.

In contrast, Keijzer et al. [24] failed to demonstrate a significant association between age at resection (before or after 2 years of age) and subsequent lung function at a mean 10 years of age. Naito et al. [25] found a preserved total lung capacity (TLC) but reduced forced expiratory volume at the first minute (FEV1) succeeding lobectomy, when assessed between 8 and 23 years of age. Age at lobectomy (before or after 2 years of age) was not significantly associated with any abnormal pulmonary function or exercise test [19].

There remains a lack of an evidence-based consensus on the management of CLM patients. Annunziata et al. [26] proposed an algorithm for the assessment and follow-up of 
congenital thoracic malformations (CTM) detected either in the antenatal or postnatal period (Fig. 2).

We adopted the early surgery principle for the fear of the risk of malignant transformation, if untreated, risk of complications, including infection and pneumothorax, and the concept of potential for compensatory lung growth with earlier resection $[19,20]$.

\section{Study limitations}

A major limitation to our study was missing data about prenatal diagnosis. The pediatric pulmonologists did not share all the cases who underwent watchful expectancy during our multidisciplinary meetings, thus none of them were included in our study.

Another limitation of our study was the few numbers of patients incorporated in the study. This was not unintentional since we needed a review of our technique in a cohort of a rare pathology and we carried a prospective study for 13 years. Other studies have shown a smaller number of patients and shorter study durations.

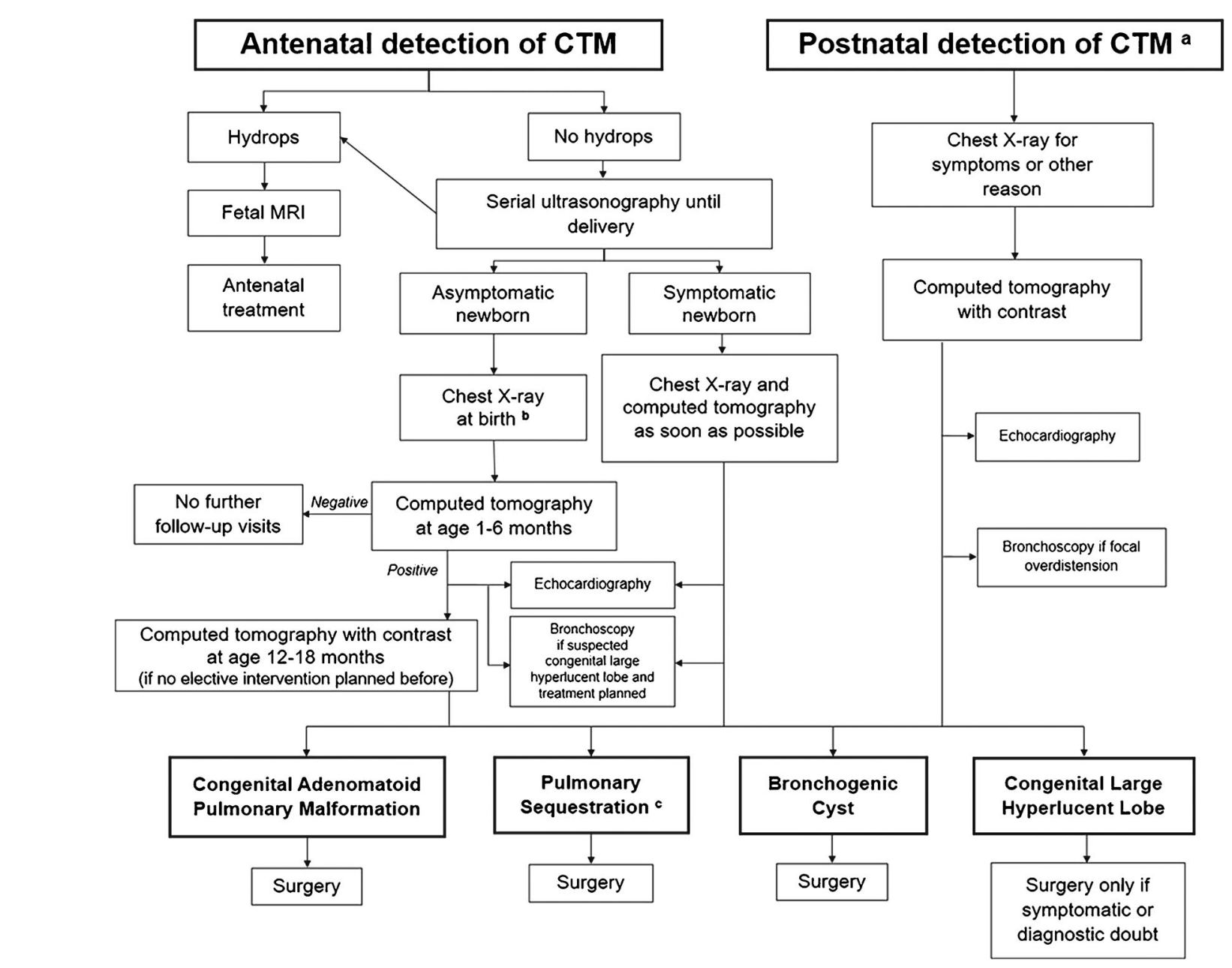

The rate of complications was high but they were comparable to other studies in the literature. The inclusion of all cases with an air leak has increased complication rates.

All the cases were operated via thoracotomy and not via video-assisted thoracoscopy, as this technology was not popular in this age at our institution at that time. Our center is now operating some pediatric patients using VATS.

\section{Conclusions}

We conclude that CLMs are rare anomalies. However, they must be kept in mind in the differential diagnosis of any pediatric case with repeated infection, respiratory distress, or having any radiological abnormalities. Surgery in the form of lobectomy or lesser resection is generally safe and the patient can be extubated at the end of the maneuver, with a reasonable postoperative course, other than some occasional treatable complications.

Fig. 2 Proposed algorithm by Annunziata et al. for follow-up of congenital lung malformations. [26] 
Acknowledgments This trial would not be possible without the enthusiasm and commitment of clinicians, nurses, and our patients.

Previous presentations Presented as a poster presentation \#1261 during the proceedings of the 26th annual meeting of the Asian Society for Thoracic and Cardiovascular Surgery (ASCVTS 18), May 2018 in Moscow, Russia.

Author contribution All authors contributed equally in conceptualization, read, and approved the manuscript in its currently submitted form.

Funding No funding was received, self-funded.

\section{Compliance with ethical standards}

Competing interests None to declare.

Ethics approval We followed the declaration of Helsinki regarding studies on human subjects. Approvals of the institutional research board of Mansoura University Faculty of Medicine was obtained. (MUIRB-15215-21). This paper does not encompass any studies with animals.

Consent to participate Patients were informed and signed written consent for participation in the study and operation. Patients rejecting the consent were not harmed as regards optimal medical care.

Consent for publication Patients were informed and signed written consent for the publishing of clinical data.

Availability of data and material The datasets used and/or analyzed during the current study available from the corresponding author on reasonable request.

\section{References}

1. Murthy RA, Kernstine KH, Burkharat HM, DeArmond DT. In: Sellke F, Del Nido PJ, Swanson SJ, editors. Congenital lung diseases Sabiston \& Spencer surgery of the chest, vol. 9; 2015. p. 151.

2. Andrade CF, Ferreira HP, Fischer GB. Congenital lung malformations. J Bras Pneumol. 2011;37:259-71.

3. Dewan RK, Kesieme EB, Sisodia A, Ramchandani R, Kesieme $\mathrm{CN}$. Congenital malformation of lung parenchyma: 15 years experience in a thoracic surgical unit. Indian J Chest Dis Allied Sci. 2012;54:105-9.

4. Zobel M, Gologorsky R, Lee H, Vu L. Congenital lung lesions. Semin Pediatr Surg. 2019;28:150821.

5. Correia-Pinto J, Gonzaga S, Huang Y, Rottier R. Congenital lung lesions - underlying molecular mechanisms. Semin Pediatr Surg. 2010;19:171-9.

6. MacSweeney F, Papagiannopoulos K, Goldstraw P, Sheppard MN Corrin B, Nicholson AG. An assessment of the expanded classification of congenital cystic adenomatoid malformations and their relationship to malignant transformation. Am J Surg Pathol. 2003;27:1139-46

7. Guo R, Zou YX, Zhai J, Zhao LS. Clinical analysis of 96 cases of congenital cystic lung lesions in children. Zhonghua Er Ke Za Zhi. 2020;58:19-24.
8. Ferreira HP, Fischer GB, Felicetti JC, Camargo Jde J, Andrade CF. Surgical treatment of congenital lung malformations in pediatric patients. J Bras Pneumol. 2010;36:175-80.

9. Costa Júnior Ada S, Perfeito JA, Forte V. Surgical treatment of 60 patients with pulmonary malformations: what have we learned? J Bras Pneumol. 2008;34:661-6.

10. Saeed A, Kazmierski M, Khan A, McShane D, Gomez A, Aslam A. Congenital lung lesions: preoperative three-dimensional reconstructed CT scan as the definitive investigation and surgical management. Eur J Pediatr Surg. 2013;23:53-6.

11. Mullassery D, Jones MO. Open resections for congenital lung malformations. J Indian Assoc Pediatr Surg. 2008;13:111-4.

12. Karunasumetta C, Kuptarnond C, Prathanee S, Intanoo W, Wongbuddha C. Surgical outcomes for congenital lung malformations: 10 years experience at a single center. J Med Assoc Thail. 2014;97:52-9.

13. Azizkhan RG, Crombleholme TM. Congenital cystic lung disease: contemporary antenatal and postnatal management. Pediatr Surg Int. 2008;24:643-57.

14. Savic B, Birtel FJ, Tholen W, Funke HD, Knoche R. Lung sequestration: report of seven cases and review of 540 published cases. Thorax. 1979;34:96-101.

15. Gezer S, Taștepe I, Sirmali M, et al. Pulmonary sequestration: a single-institutional series composed of 27 cases. J Thorac Cardiovasc Surg. 2007;133:955-9.

16. Jesch NK, Leonhardt J, Sumpelmann R, Gluer S, Nustede R, Ure BM. Thoracoscopic resection of intra-and extralobar pulmonary sequestration in the first 3 months of life. J Pediatr Surg. 2005;40: 1404-6.

17. Corbett HJ, Humphrey GM. Pulmonary sequestration. Paediatr Respir Rev. 2004;5:59-68.

18. Maier HC. Bronchogenic cysts of the mediastinum. Ann Surg. 1948; 127:476-502.

19. Hall NJ, Stanton MP. Long-term outcomes of congenital lung malformations. Semin Pediatr Surg. 2017;26:311-6.

20. Stanton M, Njere I, Ade-Ajayi N, Patel S, Davenport M. Systematic review and meta-analysis of the postnatal management of congenital cystic lung lesions. J Pediatr Surg. 2009;44:1027-33.

21. Nakajima C, Kijimoto C, Yokoyama Y, et al. Longitudinal followup of pulmonary function after lobectomy in childhood-factors affecting lung growth. Pediatr Surg Int. 1998;13:341-5.

22. Komori K, Kamagata S, Hirobe S, et al. Radionuclide imaging study of long-term pulmonary function after lobectomy in children with congenital cystic lung disease. J Pediatr Surg. 2009:44:2096100 .

23. Marshall KW, Blane CE, Teitelbaum DH, van Leeuwen $\mathrm{K}$. Congenital cystic adenomatoid malformation: impact of prenatal diagnosis and changing strategies in the treatment of the asymptomatic patient. Am J Roentgenol. 2000;175:1551-4.

24. Keijzer R, Chiu PP, Ratjen F, Langer JC. Pulmonary function after early vs late lobectomy during childhood: a preliminary study. J Pediatr Surg. 2009;44:893-5.

25. Naito Y, Beres A, Lapidus-Krol E, Ratjen F, Langer JC. Does earlier lobectomy result in better long-term pulmonary function in children with congenital lung anomalies?: a prospective study. J Pediatr Surg. 2012;47:852-6.

26. Annunziata F, Bush A, Borgia F, et al. Congenital lung malformations: unresolved issues and unanswered questions. Front Pediatr. 2019;7:239.

Publisher's note Springer Nature remains neutral with regard to jurisdictional claims in published maps and institutional affiliations. 